\title{
Ship Tracking with Static Electric Field Based on Adaptive Progressive Update Extended Kalman Filter
}

\author{
Shouwei HU ${ }^{1}$, Bing YAN 2,a \\ 1 Science and Technology on Near-Surface Detection Laboratory, Wuxi, Jiangsu 214035, China \\ ${ }^{2}$ College of Weapon Engineering, Naval University of Engineering, Wuhan, Hubei 430033, China
}

\begin{abstract}
An adaptive progressive update extended Kalman filter is introduced for unknown noise in ship tracking using static electric field. The corresponding state space model is established; the algorithm is introduced, and the simulation is designed. The simulation results show that the adaptive algorithm can effectively improve the performance of the algorithm, when the noise covariance deviates from the real value; the finite number of noise covariance estimation is beneficial to the stability of the filter.
\end{abstract}

\section{Introduction}

In recent years, ship electric field is a hot topic of research. Ship tracking with static electric field has become a new research direction, which can be an effective supplement to acoustic tracking ${ }^{[1]}$. In the tracking algorithm, extended Kalman filter (EKF) and corresponding improvement algorithm are the best choice due to their simple form, low computational complexity, and the advantage of real-time tracking. Baoquan Sun ${ }^{[2]}$ proposed a progressive update extended Kalman filter (PUEKF), which has better performance, and this article is based on the method. In Kalman filters, it is usually assumed that the statistical characteristics of environmental noise are accurately known and remain unchanged throughout the process, but in practice, environmental noise may be unknown and change. This may cause Kalman filters to lose its optimality and even cause filtering divergence. At present, in various tracking applications, scholars have put forward various methods to solve the problem of unknown statistical characteristics of environmental noise ${ }^{[3-4]}$. A noise covariance matrix estimation algorithm based on residuals is proposed in the reference[5]. The method is simple and feasible, and can make full use of the measurement information. This paper will study the adaptive Kalman filtering algorithm for ship electric field tracking based on it.

The reminder of the paper is organized as follows. In section II ship motion state space model is established. In section III, adaptive extended Kalman filter is described. In section IV, simulation to verify the performance of the method in ship tracking application is designed.

\subsection{State space model}

Ship tracking state space model contains dynamic model and measurement model. Ship movement usually has low mobility, so its dynamic model can be assumed to be linear model. Measurement model is nonlinear. The state space model of ship tracking is:

$$
\begin{aligned}
& \boldsymbol{x}_{k}=\boldsymbol{a}\left(\boldsymbol{x}_{k-1}\right)+\boldsymbol{w}_{k-1} \\
& \boldsymbol{y}_{k}=\boldsymbol{h}\left(\boldsymbol{y}_{k-1}\right)+\boldsymbol{v}_{k}
\end{aligned}
$$

Where, $\quad \boldsymbol{x}_{k} \in \mathbb{E}^{n}, \quad \boldsymbol{y}_{k} \in \mathbb{E}^{m}$ are state vector and observation vector, respectively; $\boldsymbol{w}_{k-1} \sim \mathcal{N}\left(\boldsymbol{0}, \boldsymbol{Q}_{k-1}\right)$ and $\boldsymbol{v}_{k} \sim \mathcal{N}\left(\boldsymbol{\theta}, \boldsymbol{R}_{k}\right)$ are state noise covariance and measurement noise covariance, respectively; $\boldsymbol{a}$ and $\boldsymbol{h}$ are some known functions.

\subsubsection{Measurement model}

The ship SE signal can be described by the point current array model with $N(N \geq 2)$ point currents at equal distance, in which the current density of each point current is $I_{p i}$ and the distance is $l_{d}$. The ship SE is assumed to be the sum of electric fields of the $N$ point currents $^{[6]}$

$$
U=\sum_{i=1}^{N} I_{p i} K\left(I_{p i}, P\right) .
$$

Where, $K\left(I_{p i}, P\right)$ is the distance coefficient, reflecting the function between source and field point. In the condition shown in figure 1 it is described as

\section{Problem Statement}

\footnotetext{
a Corresponding author: bingyan_wh@163.com
} 


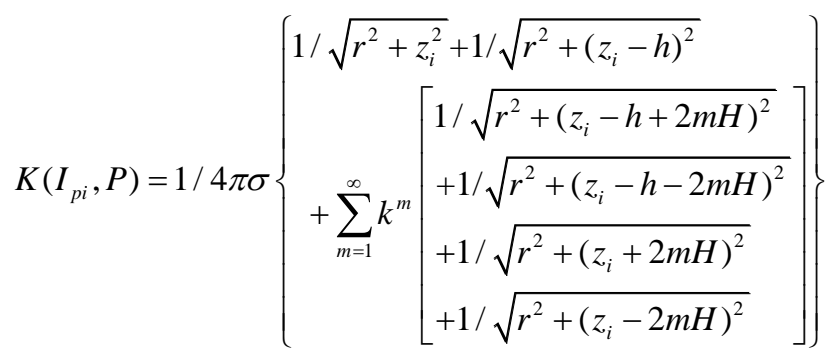

Where, $H$ is the seawater depth; $h$ is the sensor depth; $\sigma$ is the seawater conductivity; $\sigma_{1}$ is the seabed conductivity; $k=\left(\sigma-\sigma_{1}\right) /\left(\sigma+\sigma_{1}\right)$ is the submarine reflection coefficient; $m$ is the number of reflections, usually take 10 20 $0^{[6]} ; r^{2}=\left(x-x_{i}\right)^{2}+\left(y-y_{i}\right)^{2}$.

Then we can get $\boldsymbol{E}$.

$$
\begin{aligned}
& E_{x}=-\partial U / \partial x=\sum_{i=1}^{n} I_{p i} K_{1 x}\left(\mathrm{I}_{p i}, P\right) \\
& E_{y}=-\partial U / \partial y=\sum_{i=1}^{n} I_{p i} K_{1 y}\left(\mathrm{I}_{p i}, P\right) \\
& E_{z}=-\partial U / \partial z=\sum_{i=1}^{n} I_{p i} K_{1 z}\left(\mathrm{I}_{p i}, P\right)
\end{aligned}
$$

Where, $K_{1 x}\left(\mathrm{I}_{p i}, P\right), K_{1 y}\left(\mathrm{I}_{p i}, P\right), K_{1 z}\left(\mathrm{I}_{p i}, P\right)$ are the distance coefficient, reflecting the function between source and field point.

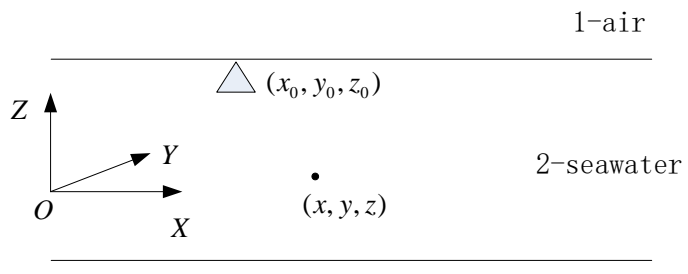

3-seabed

Figure1 Air-seawater-seabed three-layer uniform media coordinate system

The target signal measured by electric field sensor $j(j=1,2, \cdots, J)$ can be modled as follows:

$$
y^{(j)}=h^{(j)}\left(\boldsymbol{x}_{k}\right)+\boldsymbol{v}_{k}^{(j)}=\boldsymbol{E}_{k}^{(j)}+\boldsymbol{v}_{k}^{(j)}
$$

Where, $\boldsymbol{E}_{k}^{(j)}=\left[E_{k, x}^{(j)}, E_{k, y}^{(j)}, E_{k, z}^{(j)}\right]$ is signal received by electric field sensor; $\boldsymbol{v}_{k}^{(j)}=\left[v_{x, k}^{(j)}, v_{y, k}^{(j)}, v_{z, k}^{(j)}\right]$ is the background noise of the electric field sensor. The observation equation can be further obtained

$$
\boldsymbol{y}_{k}=\boldsymbol{h}\left(\boldsymbol{x}_{k}\right)+\boldsymbol{v}_{k}=\left[\begin{array}{c}
h^{(1)}\left(\boldsymbol{x}_{k}\right) \\
h^{(2)}\left(\boldsymbol{x}_{k}\right) \\
\vdots \\
h^{(J)}\left(\boldsymbol{x}_{k}\right)
\end{array}\right]+\left[\begin{array}{c}
\boldsymbol{v}_{k}^{(1)} \\
\boldsymbol{v}_{k}^{(2)} \\
\vdots \\
\boldsymbol{v}_{k}^{(J)}
\end{array}\right]
$$

\subsubsection{State model}

Ship target state vector at $k$ time is defined as follows:

$$
\boldsymbol{x}_{k}=\left[\boldsymbol{r}_{k}^{\mathrm{T}}, \boldsymbol{V}_{k}^{\mathrm{T}}, p\right]^{\mathrm{T}}
$$

Where, $\boldsymbol{r}_{k}=[x, y, z]^{\mathrm{T}}, \quad \boldsymbol{V}_{k}=\left[\boldsymbol{V}_{x}, \boldsymbol{V}_{y}\right]^{\mathrm{T}}$ (the motion in $z$ direction is neglected).
The ship target motion is modeled as a discrete white noise acceleration (DWNA) model based on the low maneuver hypothesis of the ship target. Therefore, $\boldsymbol{a}(\cdot)$ in formula(1) is a linear transformation $\boldsymbol{F}=\left[\begin{array}{cccccc}1 & 0 & 0 & T_{s} & 0 & 0 \\ 0 & 1 & 0 & 0 & T_{s} & 0 \\ 0 & 0 & 1 & 0 & 0 & 0 \\ 0 & 0 & 0 & 1 & 0 & 0 \\ 0 & 0 & 0 & 0 & 1 & 0 \\ 0 & 0 & 0 & 0 & 0 & 1\end{array}\right], T_{s}$ is the sampling interval.

The state noise covariance matrix is $\boldsymbol{Q}=\operatorname{diag}\left(\boldsymbol{\Gamma}_{m} \boldsymbol{\Sigma} \boldsymbol{\Gamma}_{m}, \alpha\right) . \boldsymbol{\Sigma}$ is the acceleration noise intensity; $\boldsymbol{\Gamma}_{m}$ is acceleration noise transformation matrix; $\Sigma=\operatorname{diag}\left(\sigma_{x}^{2}, \sigma_{y}^{2}\right) \quad \boldsymbol{\Gamma}_{m}=\left[\frac{T_{s}^{2}}{2} \mathbf{I}_{2 \times 2} ; \mathbf{0}_{1 \times 2} ; T_{s} \mathbf{I}_{2 \times 2}\right] ; \alpha$ is a small constant used to characterize the covariance of the changes in the dipole strength.

\subsection{Adaptive PUEKF}

The basic step of PUEKF is ${ }^{[2]}$ :

(1) Time update

$$
\begin{aligned}
& \boldsymbol{P}_{k \mid k-1}=\boldsymbol{F} \boldsymbol{P}_{k-1 \mid k-1} \boldsymbol{F}^{\mathrm{T}}+\boldsymbol{Q}_{k-1} \\
& \hat{\boldsymbol{x}}_{k \mid k-1}=\boldsymbol{F} \hat{\boldsymbol{x}}_{k-1 \mid k-1}
\end{aligned}
$$

(2) Measurement update

Steps1: Initialization, $\left.\hat{\boldsymbol{x}}_{0}\right|_{i=1}=\hat{\boldsymbol{x}}_{k \mid k-1},\left.\boldsymbol{P}_{0}\right|_{i=1}=\boldsymbol{P}_{k \mid k-1}$.

Steps2: $i=1,2, \cdots, N_{\mathrm{pu}}$

$$
\begin{aligned}
& \boldsymbol{S}_{i-1}=\hat{\boldsymbol{H}}_{i-1} \boldsymbol{P}_{i-1} \hat{\boldsymbol{H}}_{i-1}^{\mathrm{T}}+\boldsymbol{R}_{k} / \delta \lambda \\
& \boldsymbol{K}_{i}=\boldsymbol{P}_{i-1} \hat{\boldsymbol{H}}_{i-1}^{\mathrm{T}} \boldsymbol{S}_{i-1}^{-1} \\
& \hat{\boldsymbol{x}}_{i}=\hat{\boldsymbol{x}}_{i-1}+\boldsymbol{K}_{i}\left(\boldsymbol{y}_{k}-\boldsymbol{h}\left(\hat{\boldsymbol{x}}_{i-1}\right)\right) \\
& \boldsymbol{P}_{i}=\left(\boldsymbol{I}-\boldsymbol{K}_{i} \hat{\boldsymbol{H}}_{i-1}\right) \boldsymbol{P}_{i-1}
\end{aligned}
$$

Time subscript is omitted. $\delta \lambda$ is progressive factor.

Steps3: $\hat{\boldsymbol{x}}_{k \mid k}=\left.\hat{\boldsymbol{x}}_{1}\right|_{i=N_{\mathrm{pu}}}, \boldsymbol{P}_{k \mid k}=\left.\boldsymbol{P}_{1}\right|_{i=N_{\mathrm{pu}}}$

When $N_{\text {pu }}=1$, the PUEKF algorithm degrades to the EKF algorithm.

The residual at $k$ moment $\boldsymbol{r}_{k}$ is defined as the difference between the actual measured value $\boldsymbol{y}_{k}$ and the estimated value of the filter $\hat{\boldsymbol{y}}_{k}$.

$$
\begin{aligned}
\boldsymbol{r}_{k} & =\boldsymbol{y}_{k}-\hat{\boldsymbol{y}}_{k} \\
& =\boldsymbol{y}_{k}-\boldsymbol{H}_{k}\left(\hat{\boldsymbol{x}}_{k \mid k-1}+\boldsymbol{K}_{k}\left(\boldsymbol{y}_{k}-\boldsymbol{H}_{k} \hat{\boldsymbol{x}}_{k \mid k-1}\right)\right) \\
& =\left(\mathbf{I}-\boldsymbol{H}_{k} \boldsymbol{K}_{k}\right) \boldsymbol{d}_{k} \\
& =\left(\mathbf{I}-\frac{\boldsymbol{H}_{k} \boldsymbol{P}_{k \mid k-1}^{x x} \boldsymbol{H}_{k}^{\mathrm{T}}}{\boldsymbol{H}_{k} \boldsymbol{P}_{k \mid k-1}^{x x} \boldsymbol{H}_{k}^{\mathrm{T}}+\boldsymbol{R}_{k}}\right) \boldsymbol{d}_{k} \\
& =\boldsymbol{R}_{k} \boldsymbol{S}_{k}^{-1} \boldsymbol{d}_{k}
\end{aligned}
$$

Residual variance is: 


$$
\begin{aligned}
\boldsymbol{C}_{\boldsymbol{r}_{k}} & =\mathrm{E}\left(\boldsymbol{r}_{k} \boldsymbol{r}_{k}^{\mathrm{T}}\right) \\
& =\boldsymbol{R}_{k} \boldsymbol{S}_{k}^{-1} \boldsymbol{S}_{k} \boldsymbol{S}_{k}^{-1} \boldsymbol{R}_{k} \\
& =\boldsymbol{R}_{k} \boldsymbol{S}_{k}^{-1} \boldsymbol{R}_{k}
\end{aligned}
$$

According to the principle of Kalman filter, the gain of the filter is:

$$
\boldsymbol{K}_{k}=\boldsymbol{P}_{k \mid k-1}^{x x} \boldsymbol{H}_{k}^{\mathrm{T}} \boldsymbol{S}_{k}^{-1}=\boldsymbol{P}_{k \mid k}^{x x} \boldsymbol{H}_{k}^{\mathrm{T}} \boldsymbol{R}_{k}^{-1}
$$

Multiplied both sides of the (12) by $\boldsymbol{H}_{k}$ :

$\boldsymbol{H}_{k} \boldsymbol{P}_{k \mid k}^{x x} \boldsymbol{H}_{k}^{\mathrm{T}} \boldsymbol{R}_{k}^{-1}=\boldsymbol{H}_{k} \boldsymbol{P}_{k \mid k-1}^{x x} \boldsymbol{H}_{k}^{\mathrm{T}} \boldsymbol{S}_{\boldsymbol{k}}^{-1}=\left(\boldsymbol{S}_{\boldsymbol{k}}-\boldsymbol{R}_{k}\right) \boldsymbol{S}_{\boldsymbol{k}}^{-1}=\mathbf{I}-\boldsymbol{R}_{k} \boldsymbol{S}_{\boldsymbol{k}}^{-1}$

$\Rightarrow \boldsymbol{H}_{k} \boldsymbol{P}_{k \mid k}^{x x} \boldsymbol{H}_{k}^{\mathrm{T}}=\boldsymbol{R}_{k}-\boldsymbol{R}_{k} \boldsymbol{S}_{k}^{-1} \boldsymbol{R}_{k}$

$\Rightarrow \boldsymbol{R}_{k}=\boldsymbol{H}_{k} \boldsymbol{P}_{k \mid k}^{x x} \boldsymbol{H}_{k}^{\mathrm{T}}+\boldsymbol{C}_{r_{k}}$

So, the estimated value of $\boldsymbol{R}$ is

$$
\hat{\boldsymbol{R}}_{k}=\boldsymbol{H}_{k} \boldsymbol{P}_{k \mid k}^{x x} \boldsymbol{H}_{k}^{\mathrm{T}}+\hat{\boldsymbol{C}}_{\boldsymbol{r}_{k}}
$$

According to the windowing method, the real-time estimation variance of residual error is ${ }^{[7]}$ :

$$
\hat{\boldsymbol{C}}_{\boldsymbol{r}_{k}}= \begin{cases}\frac{k-1}{k} \hat{\boldsymbol{C}}_{\boldsymbol{r}_{k-1}}+\frac{1}{k} \boldsymbol{r}_{k} \boldsymbol{r}_{k}^{\mathrm{T}}, & k \leq \mathrm{W} \\ \frac{1}{\mathrm{~W}} \sum_{i=k-\mathrm{W}+1}^{k} \boldsymbol{r}_{k} \boldsymbol{r}_{k}^{\mathrm{T}}, \quad k>\mathrm{W}\end{cases}
$$

Where, $\mathrm{W}$ is the length of sliding data window.

The continuous change of $\boldsymbol{R}_{k}$ is actually not conducive to the stability of filtering, so it will stop the estimation of $\boldsymbol{R}_{k}$ after $N_{i}$ iterations

\section{Simulation Results And Analysis}

The root mean square errors at $k$ moment of position component $\left(R M S E_{k}^{\text {pos }}\right)$ are formulated as

$$
R M S E_{k}^{p o s}=\sqrt{\frac{1}{M C} \sum_{i=1}^{M C}\left\|\hat{r}_{k}^{(i)}-r^{(i)}\right\|_{2}^{2}} ; k=1, \cdots, T_{N}
$$

Where, $M C$ is the number of simulation.

$R M S E_{k}^{p o s}$ is took as the criterion for evaluating the algorithms

Table1 Simulation scene parameters

\begin{tabular}{cc}
\hline Parameter/(unit) & Magnitude \\
\hline $\boldsymbol{r}_{0} /(\mathrm{m})$ & {$[-100,-100,8.4]$} \\
$\boldsymbol{v}_{0} /(\mathrm{m} / \mathrm{s})$ & {$[5,5]$} \\
$\boldsymbol{I}_{0} /(\mathrm{A})$ & {$[-20,20]$} \\
$l_{d} /(\mathrm{m})$ & 10. \\
\hline
\end{tabular}

Consider a point current array composed by 2 point currents. Consider the simulation scenario in Table 1-2. In addition, the sensors are located at $(0, \pm 5,0)$; the process acceleration noise intensity $\Sigma=[0.001 ; 0.001]$, $\alpha=0.0001$; seawater depth $H=8.5 \mathrm{~m}$; progressive update factor $\delta \lambda=0.1$.

Tabel 2 Filter initial condition

\begin{tabular}{ccc}
\hline Parameter(unit) & Initial value & Initial mean square error \\
\hline$\hat{\boldsymbol{r}}_{0} /(\mathrm{m})$ & {$[-150,-200,8.5]$} & $\operatorname{diag}\left[50^{2}, 100^{2}, 0.1^{2}\right]$ \\
$\hat{\boldsymbol{v}}_{0} /(\mathrm{m} / \mathrm{s})$ & {$[1,3]$} & $\operatorname{diag}\left[2^{2}, 2^{2}\right]$ \\
$\boldsymbol{I}_{p} /(\mathrm{A})$ & {$[0,0]$} & $\operatorname{diag}[1,1]$ \\
\hline
\end{tabular}

The true observation noise covariance is $\boldsymbol{R}_{\text {true }}=\sigma^{2} \mathbf{I}$, $\sigma=1 e-5$. Set $\boldsymbol{R}_{k}=100 \times \boldsymbol{R}_{\text {true }}$. Compare the tracking effect of PUEKF and APUEKF. After 20 iterations, the estimation of $\boldsymbol{R}_{k}$ is stopped, while the previous estimate of $\boldsymbol{R}_{k}$ is adopted as a fixed value. The result is shown in Figure2.

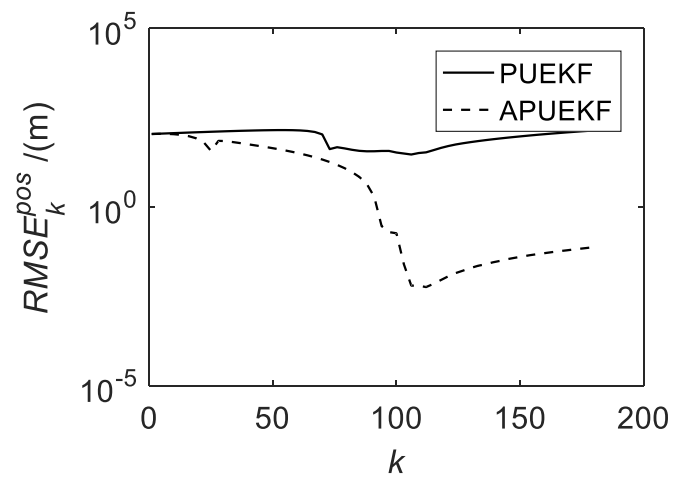

Figure2 $R M S E_{k}^{p o s}$ of the two algorithms

As shown in figure2, when $\boldsymbol{R}_{k}$ deviate from the true value $\boldsymbol{R}_{\text {true }}$, PUEKF performance slips seriously, however, APUEKF keeps good performance. Simulation result demonstrates that adaptive method can effectively improve the performance of filtering.

$N_{i}$ is changed to explore the impact of $N_{i}$ on the performance of APUEKF. Set the value of $N_{i}$ to $20,80,120,180$, the result is shown in figure3.

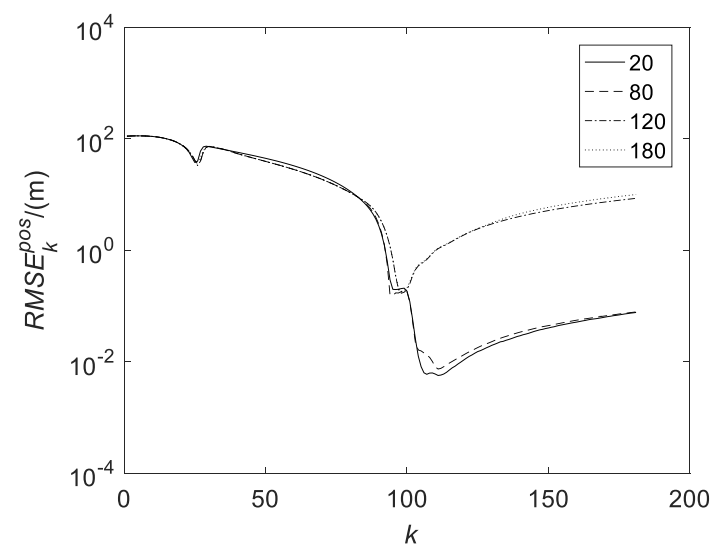

Figure3 $R M S E_{k}^{p o s}$ of APUEKF changing with $N_{i}$ 
From figure 3 we can see that it is not the bigger the $N_{i}$ is, the better. After an appropriate number of iterations, the estimation of $\boldsymbol{R}_{k}$ is stopped, which is more conducive to filter stability.

\section{Conclusions}

In this paper, an adaptive PUEKF is introduced for the unknown noise. Simulation results show that the adaptive method can effectively improve the filtering performance. In addition, the continuous change of $\boldsymbol{R}_{k}$ is actually not conducive to the stability of filtering, so it will stop the estimation of $\boldsymbol{R}_{k}$ after a certain number of iterations when environmental changes are not drastic.

\section{Funding Statement}

This study is sponsored by Science and Technology on Near-Surface Detection Laboratory fund [TCGZ2017A007].

\section{References}

1. B.Q. Sun, B. Yan, R.X. Jiang, et al. Application of Ship Static Electric Field to Ship Tracking and Positioning. Journal of Unmanned Undersea Systems, 26, 57-62 (2018).

2. B.Q. Sun, B. Yan, R.X. Jiang. A Progressive Update Extended Kalman Filter for Ship Tracking With Static Electric Field. Journal of National University of Defense Technology (to be published).

3. R.K. Mehra. Approaches to adaptive filtering. Automatic Control IEEE Transactions on, 17, 693698 (1972).

4. H.L. Mitchell. An Adaptive Ensemble Kalman Filter. Monthly Weather Review, 128, 416-433 (2000).

5. L.M. Li, W.B. Gong, H.J. Liu, et al. A carrier tracking algorithm based on adaptive extended Kalman filter. Acta Aeronautica Et Astronautica Sinica, 33, 1319-1328 (2012).

6. R.X. Jiang, Y.D. Hu, and S.G. Gong. Depth conversion of the vessel static electric field based on point charge source. Chinese Journal of Radio Science, 29, 685-693(2014).

7. V.D. Papic, Z.M. Djurovic, B.D. Kovacevic, et al. Adaptive Doppler-Kalman filter for radar systems. IEEE Proceedings Vision Image and Signal Processing, 153, 379-387(2006). 